\title{
Regulação Emocional dos Professores do II Ciclo de dois municípios de Angola em Tempo de Pandemia
}

\author{
Regulación emocional de los docentes del II Cíclo en dos municipios \\ de Angola en época de pandemia
}

Recepción del artículo: 08-09-2021 | Aceptación del artículo: 26-01-2022

Amândio Jamba Pedro da Fonseca ${ }^{1}$ amandiojamba@gmail.com

https://orcid.org/0000-0002-0135-2919

Laurindo António Tonecas ${ }^{2}$ llaurindo995@gmail.com

https://orcid.org/0000-0003-4802-9323

Ricardo Chiengo Sapalo Cassoma ${ }^{3}$ ricardocassoma@gmail.com

https://orcid.org/0000-0002-1366-5154

1Docente e Investigador do Instituto Superior Politécnico do Bié, Angola Departamento de Psicologia. Mestre em Educação Especial e Psicopedagogia 2Professor da Escola do II Ciclo do Andulo, Angola

Licenciado em Psicologia com menção em Psicologia do Desenvolvimento e da Educação ${ }^{3}$ Docente e Investigador do Instituto Superior Politécnico do Bié, Angola. Departamento de Psicologia. Mestre em Ciências da Saúde com menção em Neurociências

Para referenciar este artículo:

Pedro da Fonseca, A. J., António-Tónecas, L. y

Sapalo-Cassoma, R. C. (2022). Regulação

Emocional dos Professores do II Ciclo de dois municípios de Angola em Tempo de

Pandemia. Revista. ConCiencia EPG, 7(1),

172-186.

https://doi.org/10.32654/CONCIENCIAEPG.

$\underline{7-1.11}$

Autor corresponsal: Amândio Jamba Pedro da Fonsecaamandiojamba@gmail.com

\section{Resumo}

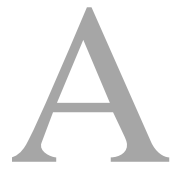

pandemia constitui problema que ativa respostas psicológicas coletivas e individuais que incidem em possível geração de respostas emocionais de inquietação ou desordem social, o que, repercute na diminuição de estabilidade física e emocional dos professores. $\mathrm{O}$ objetivo do presente estudo foi caracterizar a regulação emocional dos professores do II Ciclo dos Municípios do Andulo e Nhârea no Bié-Angola. Obedeceu a abordagem quantitativa, não experimental, transversal do tipo descritivo-comparativo. Participaram 120 professores do Andulo e Nhârea, dos quais 49 mulheres $(40,84 \%)$ e 71 homens $(59,16 \%)$, na faixa etária de 22 a 57 anos, a média de idade 34.52 anos. Os dados foram recolhidos nos meses de março a maio e, aplicou-se o Questionário de Regulação Emocional-QRE de Boian, Soares e Lima (2009); os resultados mostram que, os professores do município do Andulo utilizam mais a estratégia de reavaliação cognitiva, enquanto que, os de Nhãrea não demonstram preferências. Conclui-se que, com relação à regulação emocional por sexo, ambos utilizam mais a estratégia de reavaliação cognitiva; não há diferenças estatisticamente significativas nas médias dos indicadores estudados.

Palavras-chave: Pandemia, regulação emocional, professores. 


\section{Resumen}

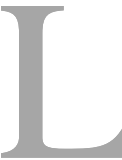

a pandemia es un problema que activa respuestas psicológicas colectivas e individuales que conducen a la posible generación de respuestas emocionales de inquietud o desorden social, lo que incide en la disminución de la estabilidad física y emocional de los profesores. El objetivo de este estudio fue caracterizar la regulación emocional de los docentes del II Ciclo de los Municipios de Andulo y Nhârea en BiéAngola. El enfoque fue cuantitativo, no experimental, transversal del tipo descriptivo-comparativo. Participaron 120 profesores de Andulo y Nhârea, 49 mujeres $(40,84 \%)$ y 71 hombres $(59,16 \%)$, con edades entre 22 y 57 años, la media de edad fue 34,52 años. Los datos fueron recolectados de marzo a mayo y se aplicó el Cuestionario de Regulación EmocionalQRE, de Boian, Soares y Lima (2009). Los resultados muestran que los docentes del municipio de Andulo utilizan más la estrategia de reevaluación cognitiva, mientras que los de Nhãrea no muestran preferencias. Se concluye que, en lo que respecta a la regulación de las emociones por sexo, ambos utilizan con mayor frecuencia la estrategia de reevaluación cognitiva; no existen diferencias estadísticamente significativas en las medias de los indicadores estudiados.

Palabras clave: Pandemia, regulación emocional, docentes.
Abstract

he pandemic is a problem that activates collective and individual psychological responses that lead to the possible generation of emotional responses of restlessness or social disorder, which affects the decrease of physical and emotional stability of teachers. The aim of this study was to characterize the emotional regulation of II Cycle teachers from the Andulo and Nhârea Municipalities in Bié-Angola. It was a quantitative, non-experimental, crosssectional descriptive-comparative approach. 120 teachers from Andulo and Nhârea participated, 49 women (40.84\%) and 71 men (59.16\%), aged between 22 and 57 years, with an average age of 34.52 years. Data were collected from March to May and it was applied the Emotional Regulation Questionnaire-QRE, of Boian, Soares y Lima (2009). The results show that teachers in the Andulo municipality use more the cognitive reassessment strategy, while Nhãrea's teachers do not show preferences. It is concluded that, with regard to emotion regulation by sex, both use more the cognitive reappraisal strategy; there are no statistically significant differences in the means of the studied indicators.

Key words: Pandemic, emotion regulation, teachers. 


\section{Introdução}

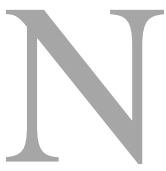

o final de 2019, um novo surto de doença de coronavírus surgiu em Wuhan, China, que rapidamente se espalhou pelo mundo, a Organização Mundial da Saúde (OMS), em março de 2020 declarou a doença como uma pandemia e chamou-a COVID-19 (Fonseca, Tur, Canhanga e Morais, 2021; Ozdede e Peker, 2020).

Em África o primeiro caso de Covid19 foi registado no dia 14 de fevereiro de 2020 no Egito, importado da China, o maior parceiro comercial de África (Fonseca et al.,2021; Gilbert et al, 2020). Até dia 12 de maio de 2021 já haviam sido notificados em todo o mundo, um total de 159.707.593 casos, 3.319.141 mortes e 446.863.658 recuperados (Johns Hopkins University, 2021).

Em Angola, o primeiro caso da COVID-19 registou-se no dia 21 de março, em Luanda, capital do país, importado de Portugal; já no dia 12 de maio de 2021 registavam-se 29.146 casos, e 639 Mortos com um total de 446.313 recuperados (CDC, 2021; Johns Hopkins University, 2021).

Mesmo assim, as aulas tiveram o seu reinício dia cinco de outubro de 2020, em todas as instituições do II Ciclo em Angola, com excepção das províncias de Luanda e Cuanza - Norte por serem as únicas afetadas pela COVID-19 naquela época com a promulgação do (Decreto Executivo no 16/2020 de 1 de outubro pp 1,2). Contudo, registaram-se incertezas no que se refere ao sucesso das atividades académicas por causa do aumento de casos positivos da
COVID-19 em todas as províncias de Angola (Fonseca et al., 2021).

Para evitar colapso no sistema de saúde, várias medidas têm sido usadas pelos governos de todos os países afetados, como por exemplo, o encerramento das fronteiras e a quarentena obrigatória, no sentido de "reduzir a curva de contaminação" e evitar colapsos nos sistemas de saúde para permitir que recebam assistência médica o maior número possível de pessoas infectadas, em Angola a situação não é diferente (Wu e McGoogan, 2020).

Desde a perspectiva psicossocial, a pandemia constitui problema que ativa respostas psicológicas coletivas que incidem seu avanço ou contenção, assim como possível geração de respostas emocionais de inquietação ou desordem social (Taylor, 2019).

As investigações sobre a regulação emocional (RE) têm aumentado substancialmente nas últimas três décadas devido sua importância para o funcionamento psicológico humano (Teixeira, Silva, Tavares e Freire, 2014). A componente RE é fundamental para um sistema saudável de relações interpessoais e está relacionada à saúde mental e ao bemestar psicossocial (Salasa, Castro, Radovic, Gross, e Turnbull, 2018).

Assim, a falta desta habilidade pode ser entendida ou associada ao desenvolvimento de problemas (Gullone, Hughes, King e Tonge, 2010; Teixeira et al. 2014). Desde a perspectiva conceptual, de acordo com os critérios de Gross e 
Thompson (2007), a RE consiste em uma ampla gama de esforços conscientes ou inconscientes realizados pela pessoa, para determinar a intensidade e a duração de suas emoções em relação às circunstâncias em que aparece, a experiência que trazem sua expressão. Na visão de Gómez e Calleja (2016), a RE é o processo pelo qual os indivíduos modulam suas emoções e modificam seu comportamento para atingir objetivos, se adaptar aos contextos ou promover o bem-estar individual e social.

Para Ehring, Tuschen-Caffier, Schunulle, Fischer e Gross (2010), a RE é a habilidade que os indivíduos possuem de ativar os mecanismos que determinam quais emoções poderão ter, quando e como irão experimentá-las e expressá-las. De acordo com, Granado, Andreu e Guiu (2014), se pode entender a RE como a modulação (aumento, retenção ou diminuição) de qualquer aspecto de uma resposta emocional, incluindo comportamento expressivo e experiência. Os processos de RE podem ser automáticos ou controlados, consciente ou inconsciente, e integram a gestão de emoções positivas e negativas (Gross e Thompson, 2007).

Os autores Morris, Silk, Steinberg, Myers e Robinson (2007), afirmam que os mecanismos reguladores emocionais incluem habilidades e estratégias que modulam as emoções específicas vivenciadas e suas dinâmicas (por exemplo, intensidade, duração e estabilidade).

Assim, compreende-se que no processo de RE utiliza-se habilidades e estratégias que possibilitam a gestão das emoções (Gouveia et al., 2018; Linhares e
Martins, 2015). Nesta linha de pensamentos, Batistoni, Ordonez, Silva, Nascimento e Cachioni (2013), salientam que, no processo de RE o indivíduo utiliza estratégias como: a) Seleção da situação, que acontece quando o sujeito opta por um contexto percebido como mais pacífico; b) Transformação da situação, quando se tenta modificar um contexto percebido como estressante para torná-lo menos ansiogênico; c) Redirecionamento do foco, quando o indivíduo mantem o foco ou atenção nos aspectos mais positivos em vez dos negativos.

No âmbito educativo, se destaca que, o desgaste dos professores está associado ao esgotamento laboral, o que se repercute na diminuição de sua estabilidade física e emocional e desgaste estão presentes em qualquer um dos níveis de ensino $\mathrm{e}$ constitui-se por três fatores principais: exaustão emocional, despersonalização ou cinismo e sensação de baixa realização individual ou profissional (Torres, 2018). Na mesma linha de pensamentos, El Sahili (2015), ressalta que a docência é uma das profissões mais propensas a sofrer síndrome de esgotamento ou insatisfação laboral, despersonalização, com mais de $9 \%$ dos professores em todo o mundo; da mesma maneira, outros profissionais vulneráveis a essa síndrome são os médicos, fisioterapeutas, psicólogos e pessoal de enfermagem, classificada como profissões potencialmente estressantes, por apresentarem carga horária excessiva.

Por esta razão, Rendón (2019), assinala que, as habilidades de RE possibilitam aos professores, a manutenção das relações interpessoais saudáveis com 
seus estudantes e outros elementos intervenientes do processo de ensino e aprendizagem, o que, favorece o ajuste pessoal, satisfação com a vida e ao bemestar emocional.

O Estado atual da RE dos professores em Angola, na província do Bié e nos municípios do Andulo e Nhãrea em particular, que desenvolvem suas atividades em tempo de pandemia da Covid-19, chama atenção pelas seguintes razões: Falta de meios de biossegurança para o combate à pandemia, falta de higienização das salas de aulas antes e depois das atividades letivas, falta de internet e aparelhos electrónicos que facilitariam o intercâmbio de materiais entre professores e alunos, existe uma alteração no funcionamento normal nos horários dos professores, pelo facto de, as turmas estarem subdividas em dois grupos, (A e B).

Por esta razão, resulta importante investigar esta temática, visto que, a literatura consultada reconhece que as competências de RE são relevantes para o bem-estar psicológico, satisfação laboral dos professores. No entanto, as questões sobre RE em professores do II ciclo no contexto angolano são pouco discutidas.

Neste sentido, o presente estudo é o primeiro que aborda acerca da RE no contexto educativo nesta região do país e, apresenta resultados importantes sobre esta temática nos professores do II ciclo em tempo de pandemia da Covid-19. Tais resultados poderão contribuir para que esta franja populacional conheça mais sobre as estratégias que podem ser utilizadas na regulação de suas emoções em diferentes situações. Também oferece um conjunto de pressupostos teóricos que sustentam a RE no âmbito educativo, o que pode enriquecer o nível de conhecimento daqueles que poderão consultar este trabalho. Assim mesmo, estes resultados poderão facilitar o Estado angolano na tomada de decisões e realizar intervenções para esta franja da população.

Por isso, justifica-se a formulação do seguinte problema de investigação: Qual é a estratégia de regulação emocional usada pelos professores do II ciclo dos Municípios do Andulo e Nhârea? Assim, o presente estudo tem como objetivo caracterizar a regulação emocional dos professores do II Ciclo dos Municípios do Andulo e Nhârea em tempo de pandemia.

\section{Método}

\section{Desenho}

Trata-se de um estudo quantitativo, não experimental, de corte transversal com alcance descritivo-comparativo, já que, este tipo de estudo mede os fenómenos e realizam análises estatísticas, seu processo é sequencial, dedutivo e analisa a realidade objetiva. Portanto, analisou-se a variável e utilizou-se a estatística para apresentar gráficos e tabelas, o fenômeno foi analisado em suas condições naturais, sem alterar ou manipular a variável. Descreveu-se quais são os elementos característicos da amostra selecionada como foco da pesquisa e comparou-se as médias segundo o sexo. Além disso, o fenômeno selecionado como objeto de estudo foi observado em um único 
momento de tempo (Hernández-Sampieri, e Torres, 2018).

\section{Participantes}

Foram selecionados 120 professores dos Municípios do Andulo e Nhârea, entre os quais 49 mulheres $(40,84 \%)$ e 71 homens (59,16\%), na faixa etária de 22 a 57 anos, com a idade média de 34,52 anos (Ver tabela 1). 0 critério de amostragem foi o não probabilístico intencional, visto que, neste tipo de amostragem todos integrantes da população não têm a mesma possibilidade de serem selecionados. Os seus procedimentos compreendem o uso de análises subjetivas, além disso este tipo de amostragem permite selecionar casos característicos da população segundo a conveniência do investigador (CossioBolaños, 2015).

\section{Tabela 1}

Características da População

\begin{tabular}{llll}
\hline Variáveis Sociodemográficas & Fi & \% \\
\hline \multirow{2}{*}{ Sexo } & Homens & 71 & 59.16 \\
& Mulheres & 49 & 40.84 \\
\multirow{2}{*}{ Idade } & 22 a 29 anos & 23 & 19.16 \\
& 30 a 40 anos & 79 & 65.84 \\
& 41 a 57 anos & 18 & 15 \\
\multirow{2}{*}{ Área de Formação } & Ciências da Educação & 84 & 70 \\
& Pré-Universitário & 2 & 1.67 \\
\multirow{2}{*}{ Local de Trabalho } & Outras & 33 & 27.5 \\
& Andulo & 93 & 77.5 \\
Nível de Formação & Nhãrea & 27 & 22.5 \\
& Médio & 8 & 6.67 \\
\hline
\end{tabular}

A seleção destes obedeceu aos seguintes critérios:

Inclusão: Professores do II Ciclo das instituições públicas dos Municípios do Andulo e Nhârea, professores que decidam participar voluntariamente na investigação por meio da assinatura do consentimento informado, professores que responderem totalmente o instrumento apresentado.

Exclusão: Professores que não apresentarem disponibilidade para participar na investigação, professores que assinarem o consentimento informado, mas que, decidam não participar na investigação, professores que não tenha respondido totalmente $\mathrm{o}$ instrumento aplicado. 


\section{Instrumento}

Para a recolecção dos dados pretende-se usar o Questionário de Regulação Emocional-QRE adaptado ao Português no contexto brasileiro por Boian, Soares y Lima (2009). 0 instrumento original está em Inglês e denomina-se Emotion Regulation Questionnaire - ERQ (Gross \& John, 2003). Este questionário baseia-se numa medida de autorrelato e está constituído por 10 itens com duas estratégias comuns pelas quais as pessoas podem regular as emoções: 4 itens avaliam a supressão das emoções e 6 medem o uso da estratégia de reavaliação das emoções. As respostas aos itens são dadas numa escala de tipo Likert que varia de $1=$ Discordo totalmente a $7=$ Concordo totalmente. Pontuações mais altas indicam o uso mais frequente de uma determinada estratégia. 0 questionário original apresenta boas propriedades com coeficiente alfa de Cronbach entre 0,79 (para a subscala de reavaliação cognitiva) e 0,73 (para a subscala de supressão emocional). Confiabilidade teste-reteste de 0,69 para ambas subscalas e análise fatorial (rotação ortogonal). Adaptou-se o instrumento dentro da compreensão e cultura angolana, através de seis expertos e para determinar o coeficiente de concordância entre eles, aplicou-se a prova de Kendall (W), cujos resultados mostraram que existe uma correlação positiva considerada alta $(\mathrm{W}=.071$. Sig. =.607). Com relação à consistência interna, demostrou um $\alpha$ 0,78 para a subscala de reavaliação cognitiva e 0,72 para a subscala de supressão emocional, na presente população.

\section{Procedimentos}

O presente estudo foi aprovado pelo Comité de Ética (ad hoc) do Instituto Superior Politécnico do Bié. Os professores selecionados como amostra da presente investigação assinaram o consentimento informado. Aplicou-se o questionário nos momentos de intervalo geral, em uma única sessão, para não afetar o normal desenvolvimento das tarefas docentes, os participantes necessitaram um tempo aproximado de 15 minutos em média para completar o questionário e utilizou-se o método tradicional de lápis e papel. A recolha de dados realizou-se de março a maio de 2021 e a análise da informação decorreu entre 24 de maio a nove de junho de 2021.

\section{Análises de dados}

Depois de obtidos os dados, foram utilizadas as planilhas do Excel 2016 e o Statistical Package for Social Sciences (SPSS24.0), para obter as medidas de tendência central e variabilidade média (M), desvio padrão (DP), frequência (Fi) e percentagem (\%). Fez-se a análise descritiva da variável de acordo com o sexo, idade, localidade, nível académico e área de formação. Para comparação das médias e verificar as diferenças segundo o sexo dos participantes, se fez o uso do teste $t$ de Studant para amostras independentes.

Utilizou-se a prova estatística de Kendall (W) para determinar o coeficiente de concordância entre os expertos e o alfa de Cronbach para a consistência interna do instrumento. 


\section{Resultados}

No indicador Reavaliação cognitiva, os resultados demostram uma média de 4,7 para a população masculina, o que indica que, esta população utiliza mais esta estratégia para regular suas emoções.

Para o indicador supressão das emoções, a média é de 3,77 os que demostra que, é uma estratégia menos utilizada, pelo facto de apresentar média relativamente inferior à reavaliação cognitiva.

Para a população feminina, o indicador reavaliação cognitiva a média é de 4,13 e para o indicador supressão das emoções a média 3,76. Tais resultados demostram que esta população utiliza a estratégia de reavaliação cognitiva. (ver tabela 2).

Ao comparar as médias do indicador reavaliação cognitiva entre homens e mulheres, foi possível encontrar que, não há diferenças estatisticamente significativas por meio do teste $\mathrm{t}(\mathrm{T}(109,631)=1,863$; $\mathrm{p}>0,05)$. Foi possível verificar que, os homens apresentam $(M=4,7 ; D P=1,24)$ e as mulheres $(\mathrm{M}=4,13 ; \mathrm{DP}=0,85)$.

Com relação ao indicador supressão emocional, também foi possível averiguar que, não há diferenças estatisticamente significativas por intermédio da prova $\mathrm{t}$ $(\mathrm{T}(104,494)=-277 ; \mathrm{p}>0,05)$. Os homens apresentam $(\mathrm{M}=3,77 ; \mathrm{DP}=0,98)$ e as mulheres $(\mathrm{M}=3,79 ; \mathrm{DP}=0,86)$.

Tabela 2

Regulação Emocional segundo sexo

\begin{tabular}{lcccc}
\hline \multirow{2}{*}{ Indicador } & \multicolumn{2}{c}{ Masculino } & \multicolumn{2}{c}{ Feminino } \\
\cline { 2 - 5 } & M & DP & M & DP \\
\hline Reavalição Cognitiva & 4,7 & 1,24 & 4,13 & 0,85 \\
Supressão das Emoções & 3,77 & 0,98 & 3,79 & 0,86 \\
\hline
\end{tabular}

No indicador a Reavaliação Cognitiva, os resultados demostram uma média de 4,34 para a população da idade compreendida entre os 22 a 29 anos, o que indica que, esta população utiliza mais esta estratégia para regular as suas emoções.

Para o indicador Supressão das emoções, a média é de 3,27 o que demostra que, é uma estratégia pouco utilizada pela população da idade compreendida entre os 22 a 29 anos, pelo facto de apresentar média relativamente inferior a Reavaliação Cognitiva.

Para a população da idade compreendida entre 30-40 anos, o indicador reavaliação cognitiva apresenta a média de 4,56.

Para o indicador supressão das emoções para a idade compreendida entre 30-40 anos a média é de 4,56 encontramos o mesmo resultado da média da 
Reavaliação Cognitiva, tais resultados demostram que esta população utiliza as duas estratégias, ou seja, não tem preferência.

Para o indicador reavaliação cognitiva, a população em idade compreendida entre 41-57 anos apresenta a média de 4,35.
Para o indicador supressão das emoções, a população em idade compreendida entre 41-57 anos demostra a média de 4,39, tais resultados demostram que esta população utiliza mais a supressão das emoções por apresentar uma média maior que a reavaliação cognitiva, conforme se observa na tabela 3 .

\section{Tabela 3}

Regulação Emocional segundo a idade

\begin{tabular}{lccccccc}
\hline \multirow{2}{*}{ Indicador } & \multicolumn{2}{c}{$\mathbf{2 2}-\mathbf{2 9}$} & \multicolumn{2}{c}{$\mathbf{3 0}-\mathbf{4 0}$} & \multicolumn{2}{c}{$\mathbf{4 1}-\mathbf{5 7}$} \\
\cline { 2 - 7 } & $\mathbf{M}$ & $\mathbf{D P}$ & $\mathbf{M}$ & $\mathbf{D P}$ & $\mathbf{M}$ & $\mathbf{D P}$ \\
\hline Reavalição Cognitiva & 4,34 & 1,10 & 4,56 & 1,11 & 4,35 & 1,32 \\
Supressão das Emoções & 4,27 & 1,06 & 4,56 & 1,11 & 4,39 & 1,35 \\
\hline
\end{tabular}

No indicador reavaliação cognitiva, os resultados demostram uma média de 4,52 para a população do Andulo, o que indica que, esta população utiliza mais esta estratégia para regular as suas emoções.

Para o indicador supressão das emoções, a média é de 4,00 o que demostra que, é uma estratégia pouco utilizada pela população do Andulo, pelo facto de apresentar média relativamente inferior a reavaliação cognitiva.

Para o indicador reavaliação cognitiva para a população da Nhârea a média é de 4,38.

0 indicador supressão das emoções para a população da Nhârea a média é de 4,38 encontramos o mesmo resultado da média da reavaliação cognitiva, estes resultados demostram que esta população utiliza as duas estratégias, conforme se observa na tabela 4 .

\section{Tabela 4}

Regulação Emocional segundo o Local de Trabalho

\begin{tabular}{lcccc}
\hline \multirow{2}{*}{ Indicador } & \multicolumn{2}{c}{ Andulo } & \multicolumn{2}{c}{ Nhârea } \\
\cline { 2 - 5 } & $\mathbf{M}$ & $\mathbf{D P}$ & $\mathbf{M}$ & DP \\
\hline Reavaliação Cognitiva & 4,52 & 1,14 & 4,38 & 1,12 \\
Supressão das Emoções & 4,00 & 0,92 & 4,38 & 1,12 \\
\hline
\end{tabular}


No indicador a reavaliação cognitiva, os resultados demostram uma média de 4,08 para a população com o Nível Académico Médio, o que indica que, esta população utiliza mais esta estratégia para regular as suas emoções

Para o indicador supressão das emoções, a média é de 3,84 o que demostra que, é uma estratégia pouco utilizada pela população do Nível Académico Médio, dado que, apresenta média relativamente inferior a reavaliação cognitiva.

Concernente o indicador reavaliação cognitiva para a população do Nível
Académico Superior a média é de 4,51, o que indica que, esta população utiliza mais esta estratégia para regular as suas emoções.

0 indicador supressão das emoções para a população do Nível Académico superior a média é de 4,50 , o que indica que, esta população utiliza menos esta estratégia, uma vez que, apresenta média relativamente inferior a reavaliação cognitiva. (Ver tabela 5).

\section{Tabela 5}

Regulação Emocional segundo o Nível Acadêmico

\begin{tabular}{ccccc}
\hline \multirow{2}{*}{ Indicador } & \multicolumn{2}{c}{ Médio } & \multicolumn{2}{c}{ Superior } \\
\cline { 2 - 5 } & $\mathbf{M}$ & DP & M & DP \\
\hline Reacaliação Cognitiva & 4,08 & 0,94 & 4,51 & 1,14 \\
Supressão das Emoções & 3,84 & 1,02 & 4,50 & 0,93 \\
\hline
\end{tabular}

Com relação ao indicador reavaliação cognitiva, os resultados demostram uma média de 4,54 para a população com a formação em Ciências da Educação, o que indica que, esta população utiliza mais esta estratégia para regular as suas emoções.

Para o indicador supressão das emoções, a média é de 3,76 o que demostra que, é uma estratégia menos utilizada pela população com a formação para a área de Ciências da Educação, pelo facto de apresentar média relativamente inferior a reavaliação cognitiva.
Para o indicador reavaliação cognitiva para a população com a formação pré-universitária a média é de 4,75 , o que indica que, esta população utiliza menos esta estratégia para regular as suas emoções, uma vez que, apresenta uma média relativamente baixa em relação a supressão das emoções.

No indicador supressão das emoções para a população com a formação préuniversitária apresenta a média de 5,13, o que indica que, esta população utiliza mais esta estratégia, pelo facto de apresentar média relativamente superior a reavaliação cognitiva. 
Concernente o indicador reavaliação cognitiva, os resultados demostram uma média de 4,35 para a população formada em outras áreas, o que indica que, esta população utiliza mais esta estratégia no momento de regular as suas emoções.
Para o indicador supressão das emoções, a média é de 3,75 o que demostra que, é uma estratégia pouco utilizada pela população formada em outras áreas, pelo facto de apresentar média relativamente inferior a reavaliação cognitiva. (ver tabela 6).

\section{Tabela 6}

Regulação Emocional segundo a Área de Formação

\begin{tabular}{lcccccc}
\hline \multirow{2}{*}{ Indicador } & \multicolumn{2}{c}{ Ciência da Educação } & \multicolumn{2}{c}{ Pré-Universitário } & \multicolumn{3}{c}{ Outras Áreas } \\
& M & DP & M & DP & M & DP \\
\hline Reavalição Cognitiva & 4,54 & 1,16 & 4,75 & 1,30 & 4,35 & 1,09 \\
Supressão das Emoções & 3,76 & 0,92 & 5,13 & 0,88 & 3,75 & 0,92 \\
\hline
\end{tabular}

\section{Discussão}

Atualmente, analisar a regulação emocional dos professores em tempo de pandemia constitui-se tema relevante para as investigações da área da educação. Para Justo e Andretta (2020), os professores são uma classe de altos índices de enfermidades psicológicas devido a sua profissão.

De acordo com os resultados, os professores de ambos os sexos utilizam mais a estratégia de reavaliação cognitiva do que a de supressão emocional. Resultados contraditórios foram encontrados por Morris et al. (2007), os autores sugerem que, normalmente, as mulheres regulam melhor as emoções cognitivamente do que os homens, este facto, pode ser atribuído a diferenças inatas nos níveis de reatividade.

Os resultados da presente investigação, demostram que, não há diferenças estatisticamente significativas por meio do teste $t$ de Studant, nas médias dos indicadores reavaliação cognitiva e supressão emocional.

Esta investigação, demostra que, os professores nas faixas etárias dos 22 aos 29 e dos 41 aos 57 anos utilizam mais a estratégia de reavaliação cognitiva. Estes resultados levam em crer que, a idade pode também, ter efeitos na qualidade da regulação emocional. Deste modo, Gross, Carstensen, Tsai, Skorpen e Hsu (1997), desenvolveram um estudo virado principalmente na análise das diferenças de idade concernente à experiência, expressão e ao controlo emocional. Os participantes apresentaram a idade compreendida entre os 19 e os 101 anos. Os resultados encontrados nesta investigação mostraram um padrão consistente atribuído às diferenças de idade. 
Neste mesmo sentido, a investigação realizada por MacDermott et al. (2010), ressaltou que, os mais jovens a frequentarem o ensino primário, apresentou um valor superior de regulação emocional, em relação aqueles que frequentavam o ensino secundário. No entanto, diante de tais resultados, parecenos importante afirmar que, se justifica um maior investimento teórico e empírico na questão de compreender a relação entre o género e a idade na regulação das emoções.

Com relação aos professores da faixa etária dos 30 aos 40 anos não têm preferências, ou seja, utilizam as duas estratégias na mesma proporção, dado que, apresentam médias similares. Resultados idênticos foram encontrados por Batistoni et al. (2011), os autores, em seu estudo no Brasil, ressaltam que, as pessoas de maior idade não revelam a tendência de uso exclusivo de uma ou outra estratégia. Nesta perspectiva, John e Gross (2004), assinalam que, as estratégias não podem ser compreendidas principalmente como boas ou más, dado que, seu uso está em dependência da situação ou contexto, da experiência emocional, assim como, de outras variáveis implicadas no desenvolvimento, tais como os mecanismos de optimização, seleção e compensação cujo resultado é uma resposta mais assertiva aos recursos emocionais disponíveis, facto que, não exclui a supressão das emoções.

Os resultados deste estudo relatam que, os professores do município do Andulo utilizam mais a estratégia de reavaliação cognitiva, enquanto que, os do município de Nhãrea não demostram preferências, dado que, as duas estratégias (reavaliação cognitiva e supressão emocional) apresentam a mesma média $(M=4,38)$.

Segundo os resultados por nível académico, todos os professores, no momento de regular suas emoções em tempo da pandemia da Covid-19 em Angola, utilizam mais a estratégia de reavaliação cognitiva. Estes resultados, levam em acreditar que, a supressão emocional considera-se disfuncional em tempos de crise, devido aos efeitos das emoções negativas. No mesmo sentido, Gondim et al. (2015); Gouveia et al. (2018), afirmam que, a supressão das emoções parece configurada como uma estratégia desadaptativa ou disfuncional, maximizando os efeitos prejudiciais das emoções negativas, ou seja, no processo de regulação emocional, evita-se expressar afetos positivos, ou mesmo negativos das emoções.

Concernente a área de formação, os resultados assinalam que todos os professores utilizam mais a estratégia de reavaliação cognitiva do que a supressão das emoções. Tais resultados permitem assegurar que, embora seja difícil controlar as emoções, por revelarem os nossos sentimentos em diversos contextos, estes professores conseguem controlá-las e evidenciar a racionalização.

\section{Limitações}

Existem algumas limitações que devem ser tomadas em conta na hora de interpretar estes resultados. 0 questionário é de autorrelato, o implica dizer que, os participantes podem dar respostas que não 
são as que realmente praticam; a amostra é intencional, o que não permite generalizar os resultados; a falta de controlo de algumas variáveis sociodemográficas, como a formação contínua dos professores e o tempo de serviço, que são elementos importantes para o desempenho desta clase.

\section{Conclusões}

$\mathrm{Na}$ presente investigação afirmou-se que, desde a perspectiva psicossocial, a pandemia constitui problema que ativa respostas psicológicas coletivas e individuais que incidem em possível geração de respostas emocionais de inquietação ou desordem social.

Com relação à regulação emocional por sexo, conclui-se que, os professores de ambos os sexos utilizam mais a estratégia

\section{Referências}

Batistoni, S. S. T., Ordonez, T. N., Silva, T. B. L., Nascimento, P. P. P., y Cachioni, M. (2013). Emotional Regulation Questionnaire (ERQ): Indicadores Psicométricos e Relações com Medidas Afetivas em Amostra Idosa. Psicologia, Reflexão e Crítica, 26, 10 18.

https://doi.org/10.1590/S010279722013000100002.

Centres for Diseases Control and Prevention (12th May, 2021). Africa CDC updates on COVID-19. https://africacdc.org/covid-19/ May 12th.[Accessed, 2021]

Cossio-Bolaños, M. (2015). Métodos de investigación cuantitativa en de reavaliação cognitiva. Não há diferenças estatisticamente significativas por meio do teste $\mathrm{t}$ de Studant, nas médias dos indicadores reavaliação cognitiva e supressão emocional.

Os professores do município do Andulo utilizam mais a estratégia de reavaliação cognitiva, enquanto que, os de Nhãrea não demostram preferências, dado que, as duas estratégias (reavaliação cognitiva e supressão emocional) apresentam a mesma média $(M=4,38)$.

Os professores das faixas etárias dos 22 aos 29 e dos 41 aos 57 anos utilizam mais a estratégia de reavaliação cognitiva, enquanto que, os da faixa etária dos 30 aos 40 anos não têm preferências, ou seja, utilizam as duas estratégias na mesma proporção.

Ciencias de la Educación. Talca, Chile: Universidad Católica del Maule.

Decreto Executivo no 16/2020 de 1 de Outubro Pg 1,2

Ehring, T., Tuschen-Caffier, B., Schnülle, J., Fischer, S., \& Gross, J. J. (2010). Emotion regulation and vulnerability to depression: Spontaneous versus instructed use of emotion suppression and reappraisal. Emotion, 10, 563-572. DOI: $10.1037 / \mathrm{a} 0019010$

El Sahili, L. (2015). Burnout. Consecuencias y soluciones. Manual Moderno. 
Fonseca, A. J. P., Tur, R. G., Canhanga, A. P. E., e Morais, M. F. (2021). Ansiedade, Depressão e Estresse do estudantado universitário pelo reinício das aulas em tempo de pandemia. Revista Actualidades Investigativas en Educación, 21(3), 1-22. 10.15517/aie.v21i3.46439.

Gilbert, M., Pullano, G., Pinotti, F., Valdano. E., Poletto, C., Boëlle, P.-Y., D'Ortenzio, E., Yazdanpanah, Y., Eholie, S. P., Altmann, M., Gutierrez, B., Kraemer, M. U. G., \& Colizza, V. (2020). Preparedness and vulnerability of African countries against importations of COVID-19: a modelling study. https://doi.org/10.1016/S01406736(20)30411-6.

Gómez, 0., Calleja, N. (2016). Regulación emocional: definición, red nomológica y medición. Revista Mexicana de Investigación en Psicología, 8(1), 96-117.

Gouveia, V. V., Moura, H. M., Oliveira, I. C. V., Ribeiro, M. G. C., Rezende, A. T., \& Brito, T. R. S. (2018). Emotional Regulation Questionnaire (ERQ): Evidence of Construct Validity and Internal Consistency. Psico-USF, Bragança Paulista, 23(3), 461-471. https://doi.org/10.1590/141382712018230306.

Granado, X. O., Andreu, M. G., y Guiu, G. F. (2014). Regulación emocional de los resultados adversos en competición. Estrategias funcionales en deportes colectivos.
Cuadernos de Psicología del Deporte, 14(1), 63-72.

Gross, J.J., \& Thompson, R. (2007). Emotion Regulation: conceptual foundations. In J.J. Gross (Eds.), Handbook of emotion regulation (pp. 3-24). New York: The Guildford Press.

Gullone, E., Hughes, E. K., King, N. J., \& Tonge, B. (2010). The normative development of emotion regulation strategy use in children and adolescents: A two year follow-up study. Journal of Child and Psychology and Psychiatry, 51(5), 567-574. 10.1111/j.14697610.2009.02183.x.

Hernández-Sampieri, R., e Torres, C. P. M. (2018). Metodología de la Investigación Científica: Las rutas cuantitativa, cualitativa y mista. McGraw-Hill.

Johns Hopkins University. (2021). Coronavirus COVID-19 Global Cases by the Center for System Science and Engineering (CSSE). https://coronavirus. jhu.edu/map.html. [Accessed May 12th, 2021]

Linhares, M. B. M., \& Martins, C. B. S. (2015). 0 processo de autorregulação no desenvolvimento de crianças. Estudos de Psicologia, 32, 281-293. 10.1590/0103166X2015000200012

Morris, A. S., Silk, J. S., Steinberg, L., Myers, S. S., \& Robinson, L. R. (2007). The role of the family context in the development of emotion regulation. 
Social Development, 16(2), 361-388. doi:10.1111/j.1467-

9507.2007.00389.x.

Ozdede, M., \& Peker, I. (2020). Analysis of Dentistry YouTube Videos Related To COVID-19. Brazilian Dental Journal, 31(4): 392-398 http://dx.doi.org/10.1590/01036440202003767

Rendón, M. A. (2019). Competencias socioemocionales de maestros en formación y egresados de programas de educación. Praxis \& Saber, 10(24), 243-270. https://doi.org/10.19053/221601 59.v10.n25.2019.10004.

Salasa, C. E., Castro, O., Radovic, D., Gross, J. J., \& Turnbull, O. (2018). The Role of Inner Speech in Emotion Dysregulation and Emotion Regulation Strategy Use. Revista Latinoamericana de Psicología, 50(2), 79-88. Doi: http://dx.doi.org/10.14349/rlp.20 18.v50.n2.1.

Taylor, S. (2019). The Psychology of Pandemics: Preparing for the Next Global Outbreak of Infectious Disease. Cambridge Scholars Publishing.

Teixeira, A., Silva, E., Tavares, D., \& Freire, T. (2014). Portuguese validation of the
Emotion Regulation Questionnaire for Children and Adolescents (ERQCA): relations with self-esteem and life satisfaction. Child Ind Res, 1-17. 10.1007/s12187-014-9266-2.

Torres, E. (2018). Competencias socioemocionales y creencias de autoeficacia como predictores del burnout en docentes mexicanos. REXE: Revista de estudios $y$ experiencias en educación, 17(35), $15-$

27. doi.org/10.21703/rexe.20181735t orres1.

Wu, Z., \& McGoogan, J. M. (2020). Characteristics of and important lessons from the coronavirus disease 2019 (COVID-19) outbreak in China: Summary of a report of 72 314 cases from the Chinese Center for Disease Control and Prevention. Journal of the American Medical Association, 323(13), 1239-1242 